Research Paper

\title{
Downregulation of promoter methylation gene PRDM5 contributes to the development of tumor proliferation and predicts poor prognosis in gastric cancer
}

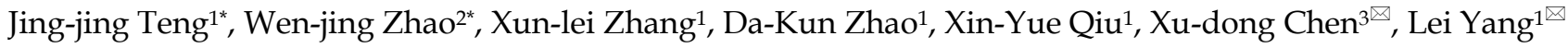 \\ 1. Department of Oncology, Affiliated Tumor Hospital of Nantong University, No.30 Tongyang North Road, Nantong 226361, China. \\ 2. Cancer Research Center Nantong, Tumor Hospital Affiliated to Nantong University, Nantong. \\ 3. Department of Pathology, Tumor Hospital Affiliated to Nantong University, Nantong, China. \\ *These authors contributed equally to this work.
}

$\triangle$ Corresponding authors: Lei Yang, E-mail: yanglei@ntu.edu.cn; Department of Oncology, Tumor Hospital Affiliated to Nantong University, Nantong, China, Jiangsu 226361, China. Xudong Chen, chenxudong@ntu.edu.cn ,Department of Pathology, Tumor Hospital Affiliated to Nantong University, Nantong, 226361, China.

(c) The author(s). This is an open access article distributed under the terms of the Creative Commons Attribution License (https://creativecommons.org/licenses/by/4.0/). See http://ivyspring.com/terms for full terms and conditions.

Received: 2021.03.02; Accepted: 2021.09.07; Published: 2021.10.03

\begin{abstract}
Background: Epigenetic aberrations of tumor suppressor genes (TSGs), particularly DNA methylation, are frequently involved in the pathogenesis of gastric cancer (GC). Previous studies have shown that PRDM5 is methylated and silenced in GC. However, the role of PRDM5 in GC progression has not been explored.

Methods: The expression and epigenetic alterations of PRDM5 in GC were analyzed in public datasets. The mRNA and protein expression of PRDM5 in fresh tissues were detected by semi-quantitative PCR and Western blot. And expression of PRDM5 in gastric paracarcinoma and carcinoma tissues from 162 patients was detected by immunohistochemistry (IHC) and assessed the association with different clinicopathological features. The prognostic value of PRDM5 in GC patients was evaluated using KaplanMeier plotter. We also studied promoter region methylation of PRDM5 in GC by methylation-specific PCR (MSP). The effects of PRDM5 on cell proliferation and migration were conducted by functional experiments in vitro.

Results: The expression of PRDM5 was downregulated in GC, and that was associated with poor survival and tumor progression. And PRDM5 expression was found to be an independent prognostic factor for GC. We also found that the methylation of PRDM5 promoter was closely related to the histopathological types and the progression of tumors through the public relations database. In vitro, ectopical expression of PRDM5 inhibited the growth of tumor cells, while knockdown of PRDM5 increased the proliferation and migration of tumor cells.

Conclusion: These results suggest that PRDM5 may be a novel TSG methylated in GC that plays important roles in GC development. And we found PRDM5 as a potential survival biomarker for GC, especially in well differentiated GC. PRDM5 expression was significantly correlated with tumor stage and histological type.
\end{abstract}

Key words: GC; Promoter methylation; PRDM5; proliferation; prognosis

\section{Introduction}

Gastric cancer remains an important cancer worldwide and it is the fifth most frequently diagnosed cancer and the third leading cause of cancer death. In particular, the incidence of GC is the highest in East Asia [1]. In the past few decades, comprehensive utilization of various treatment methods has significantly improved the survival of patients with early GC, but the prognosis of advanced GC did not significantly improve [2]. When GC is mostly diagnosed as advanced, the prognosis of 
advanced GC is usually poor, with a 5-year overall survival rate of less than $20 \%$ [3]. Therefore, a better understanding of the pathogenesis and molecular events of GC can help us to establish new diagnostic techniques, treatment regimens and prevention strategies for this disease.

The occurrence of GC involves the accumulation of various genetic and epigenetic changes, which leading to the activation of oncogene and the inactivation of tumor suppressor [1]. An increasing body of evidence indicates that in addition to changes in DNA sequence, epigenetic alterations contribute to GC initiation and progression [2], including DNA methylation of $\mathrm{CpG}$ islands, post-translational modifications of histones, microRNAs, noncoding RNAs, and nucleosome positioning [3]. DNA methylation is the first epigenetic marker that has been proved to be closely related to tumorigenesis, and it provides a stable gene silencing mechanism. DNA methylation is associated with histone modification and other chromatin related proteins and plays an important role in regulating gene expression and chromatin structure [4]. So far, a large number of genes with different biological functions have been found to be methylated in GC. Among them, PRDM5 was found to be frequently methylated and silenced in gastric cancer [5].

PRDM5 (also known as PFM2) is a member of the PRDM gene family and is usually located in the region 4q25-26 [6]. The PRDM5 protein was first identified from an EST database based on its N-terminal conserved PR domains, which typically have sequence-specific DNA binding activity [7]. Epigenetic silencing of PRDM5 is a common event in GC, and it has been demonstrated that this silencing of PRDM5 is mediated by DNA methylation or H3K27 trimethylation [8]. Moreover, methylation of PRDM5 can be detected in gastric juice of early GC [9], this suggests that PRDM5 is expected to be a good indicator for the diagnosis of GC. PRDM5 has been shown to inhibit tumor growth in vitro in patients with advanced GC, but its specific clinical significance and the critical roles in GC have rarely been probed.

In this study, we verified that the silencing of PRDM5 in GC was due to the methylation of its promoter, and confirmed its tumor suppressive effect in GC. We further explored the clinical significance of PRDM5, suggesting that the silencing of PRDM5 expression is closely related to clinical staging of GC and can indicate a poor prognosis.

\section{Materials and methods}

\section{Public cancer database analysis}

The expression analysis of PRDM5 in GC specimens was based on data retrieved from The Cancer Genome Atlas (TCGA) data portal (https:// portal.gdc.cancer.gov/). R software (http://www.rproject.org/) was applied to extract and normalize the mRNA expression data from these databases, and then the statistical analyses were performed using GraphPad Prism software version 8.0 (GraphPad Software Inc.). To explore the molecular mechanism contributing to the aberrant expression of PRDM5 in gastric cancer, the correlations between mRNA expression of PRDM5 and DNA methylation were analyzed using online database MEXPRESS (https:/ / mexpress.be) [10].

\section{Tumor samples}

This study was approved by the Medical Ethics Committee of the Affiliated Tumor Hospital of Nantong University. A total of 162 formalin-fixed paraffin-embedded (FFPE) specimens and fresh surgical tissue samples were used in this study. After the review and approval of the Ethics Committee of the Affiliated Cancer Hospital of Nantong University, 162 patients with gastric cancer who were treated in the hospital from January 2007 to December 2013 were selected as the research objects. Tissue specimens of all selected cases were selected according to the following criteria: 1) The selected cases were based on relevant imaging diagnostic data; 2) All patients had clear postoperative pathological diagnosis; 3) No preoperative treatment, including chemoradiotherapy, targeted therapy and immunotherapy; 4) Classification according to WHO (2008) standards; 5) All enrolled gastric cancer patients had complete and detailed clinicopathological data, and the follow-up time was up to 2020-08-12. The tissue samples were collected and made into tissue microarray. Specifically, the specimens were GC tissue and paired adjacent normal tissues $(>5 \mathrm{~cm}$ from the corresponding tumor edge). No patient received any adjuvant therapy before surgery. Clinicopathological information was also collected. Written informed consent was acquired from all human participants after complete description of the study. All procedures performed in studies involving human participants were in accordance with the ethical standards of the institutional and/or national research committee and with the 1964 Helsinki Declaration and its later amendments or comparable ethical standards.

\section{Immunohistochemistry}

In the immunostaining of the tissue microarray (TMA), the primary antibody was anti-PRDM5 antibody (1:300 dilution, number: 7D4C12, NOVUS). The specific experimental procedures refer to the 
previous literature [11]. The pathologists who were unaware of the patient's data evaluated the two points selected by the same patient's gastric cancer tissue. When the scores were inconsistent, the original wax block of the patient was found to be re-scored to determine the final scoring results. Scoring rules: The percentage of positive cells in each field was used as the scoring basis, that is, the score was $0-100$ points. During the analysis, we determined that the score less than 25 was divided into the low-expression group, and the score greater than or equal to 25 was divided into the high-expression group.

\section{Cell culture and transfection}

We selected three gastric adenocarcinoma cell lines (AGS, SGC-7901, and HGC-27), among which HGC-27 is an undifferentiated low-adhesion GC cell. They are provided from Center Laboratory of Tumor Hospital affiliated to Nantong University. All cell lines were grown in RPMI-1640 supplemented with $10 \%$ FBS (Invitrogen, Carlsbad, CA) at $37^{\circ} \mathrm{C}$ and $5 \%$ $\mathrm{CO}_{2}$. Small interfering RNAs (siRNA) targeting the PRDM5 transcript were purchased from ObiO Technology. Sequences were $\left(5^{\prime}-3^{\prime}\right)$ : AGCUAAAACG UCAUAUGAUTT and AUCAUAUGACGUUUUAG CUTT. The siRNA transfection was performed using Lipofectamine 2000 (Thermo Fisher Scientific, Inc., Waltham, MA, USA) according to the manufacturer's instructions. The culture medium was replaced following a 4-h transfection. After $48 \mathrm{~h}$, the cells were harvested for subsequent experiments. For PRDM5 knockdown, the cells were transfected with $50 \mathrm{nM}$ specific PRDM5 siRNAs or scrambled siRNA using serum-free Opti MEM (from Thermo Scientific). Small activating RNAs (saRNA) targeting the PRDM5 transcript were purchased from Shanghai Genechem. Sequences were $\left(5^{\prime}-3^{\prime}\right)$ : GCCCGGATCCGTTCCTGC CAT and ATGGCAGGAACGGATCCGGGC. The specific experimental process is completed by referring to the product manual.

\section{Cell proliferation assay and colony formation assays}

Treated cells $\left(1 \times 10^{6}\right.$ for AGS and SGC7901) were seeded in triplicate into 96-well plates and allowed to incubate for $24 \mathrm{~h}, 48 \mathrm{~h}$ and $72 \mathrm{~h}$. Cell viability was assessed according to the Cell Counting Kit-8 (CCK-8) protocol (Dojindo, Kumamoto, Japan). For colony formation, treated GC cells were seeded in 6-well plates at 200 cells per well [23]. Two weeks later, colonies were fixed with $4 \%$ paraformaldehyde and stained with $0.5 \%(\mathrm{~W} / \mathrm{W})$ crystal violet (diluted in phosphate buffer saline, PBS) for $30 \mathrm{~min}$.

\section{Cell migration assays}

Transwell cell migration assays were performed using boyden chambers with a polycarbonate Nucleopore membrane. $5 \times 10^{4}$ cells in $200 \mu \mathrm{l}$ serumfree Dulbecco's modified Eagle's medium were placed in the upper part of each chamber and the lower compartments were filled with $500 \mu \mathrm{l}$ Dulbecco's modified Eagle's medium containing 10\% serum as described previously.

\section{Western blot analysis}

Total protein was isolated from the tissues by using RIPA buffer; $100 \mu \mathrm{g}$ protein were loaded and separated by $10 \%$ SDS-PAGE and transferred to PVDF membranes (Millipore, Billerica, MA, USA). Next, the membranes were incubated with specific antibody for PRDM5 (Abcam, 1:1500) or GAPDH (Abcam, 1:5000) at $4{ }^{\circ} \mathrm{C} \mathrm{o} / \mathrm{n}$. The membranes were washed and then incubated with secondary antibody for $2 \mathrm{~h}$ at room temperature. Finally, the membranes were developed using ECL kit (Pierce, Rockford, IL, USA) and exposed to X-ray film for analysis by Image.lab3.0 software.

\section{qRT-PCR}

Total RNA was extracted from the tissues using TRIzol (Invertrogen, USA) following the manufacturer's manual. cDNA was synthesized by reverse transcription using RT kit (Promega, Madsion, WI, USA) following the manufacturer's manual. PCR was performed with Taq Master Mix (Promega, Madison, WI, USA) with the primers: PRDM5 GATCAAGTGGGTGCTCACAA and CATTGATAGG GACGCTCACC, product $474 \mathrm{bp}$, CAC GATGGAGG GGCCGGACTCATC and TAA AGACCTCTATGCC AACACAGT, product 225 bp. Amplification conditions were as follows: $5 \mathrm{~min}$ at $94^{\circ} \mathrm{C}$ (one cycle); $30 \mathrm{~s}$ at $94^{\circ} \mathrm{C}, 30 \mathrm{~s}$ at $58^{\circ} \mathrm{C}$, and $30 \mathrm{~s}$ at $72^{\circ} \mathrm{C}(35$ cycles $)$ and $72{ }^{\circ} \mathrm{C}$ for $5 \mathrm{~min}$ (one cycle).

\section{Methylation-specific PCR (MSP)}

Genomic DNA was extracted from the tissues using Universal Genomic DNA Extraction Kit (Takara, Tokyo, Japan). Genomic DNA (1 $\mu \mathrm{g})$ was modified with sodium bisulfite using EZ-DNA methylation kit (Zymo research, Orange, CA, USA). Bisulfite-treated DNA was used for methylationspecific PCR (MSP). MSP primers were designed by online software (http://www.urogene.org/ methprimer/index1.html). The primers sequence were as follows:

- Methylation primer: TTTTATAGGGAGTAATG GTTTAGCG and GCTAATTAACCCGAAATTA ACGAC;

- Unmethylation primer: TTTATAGGGAGTAAT GGTTTAGTGG and CACTAAT TAA CCCAAA ATTAACAAC. 
PCR amplification system $(25 \mu \mathrm{l})$ includes $10 \times$ Buffer $2.5 \mu \mathrm{l}$, dNTP $1.0 \mu \mathrm{l}, 1 \mu \mathrm{l}$ each methylation or unmethylation primers, DNA template $2 \mu \mathrm{l}$, and $\mathrm{MgCl}_{2} 2 \mu \mathrm{l}$. PCR parameters include $95^{\circ} \mathrm{C}$ for $5 \mathrm{~min}$, then $95^{\circ} \mathrm{C}$ degeneration for $30 \mathrm{~s}$, annealing for $30 \mathrm{~s}$, and $72{ }^{\circ} \mathrm{C}$ extensions for $30 \mathrm{~s}$. PCR products were electrophoresed on $2 \%$ agarose gel, and the images were scanned using the UV gel imaging system.

\section{Statistical analysis}

The statistical analyses were performed using GraphPad Prism software version 8.0 (GraphPad Software Inc.) and SPSS version 25.0 (SPSS Inc.). Differences between groups were determined using the Chi-square test or Student's t test. Overall survival (OS) after surgery was calculated using the KaplanMeier method. Cox stepwise multivariate regression analysis of prognostic factors was performed. A p value of less than 0.05 was considered to be statistically significant.

\section{Results}

\section{PRDM5 expression is frequently decreased in GC}

Through analysis of PRDM5 expression in public datasets from GC patients, we found that PRDM5 was remarkably downregulated in GC tissues compared to normal tissues (Fig. 1A, B). To further verify this phenomenon, we randomly selected 8 fresh tumor tissues and paired adjacent normal tissues of GC patients for the detection of mRNA levels and protein levels. We found that The expression of PRDM5 in gastric cancer tissues was lower than that in paracancer tissues, and only a few GC tissues showed no significant change in the expression of PRDM5 (Fig. 1C, D).

\section{Low expression of PRDM5 was associated with clinical stage and was an independent prognostic factor for gastric cancer}

To evaluate the correlation between PRDM5 expression and patients' survival, we observed RPDM5 expression that performed IHC and analyzed in 162 gastric cancer patients. According to the results of immunohistochemistry, we found that PRDM5 was generally expressed in cytoplasm, but the expression in nucleus was different. In the nucleus of gastric cancer cells, PRDM5 is rarely expressed, while in the nucleus of normal tissues adjacent to cancer, PRDM5 is almost always expressed. Representative results are shown in Fig. 2A-C. The correlations between PRDM5 protein expression and the clinicopathologic parameters of GC patients are summarized in Table 1. In GC, PRDM5 protein expression was significantly correlated with clinical stage $(p=0.036)$. Kaplan-Meier curves for overall survival (OS) were analyzed. Patients with high PRDM5 expression had significantly better survival $(p=0.031)$ (Figure 2D). Interestingly, we found that low PRDM5 expression in well-differentiated GC tissues suggested poor prognosis (Figure 2E), while no significant difference in prognosis was observed in poorly differentiated patients (Figure 2F). Furthermore, Univariate and multivariate Cox regression analyses were performed to identify important prognostic factors of OS (Table 2). PRDM5 expression $(p=0.034)$, tumor diameter $(p<$ $0.001)$, clinical stage $(p<0.001)$ and vascular invasion $(\mathrm{P}=0.048)$ were identified as important risk factors for OS. In multivariate Cox analysis, PRDM5 expression $(p=0.043)$, tumor diameters $(p=0.024)$ and clinical stage $(p<0.001)$ were found to be independent prognostic factors for OS. These findings indicate that high expression of PRDM5 could predict good prognosis in patients with GC, especially in patients with well differentiated GC.

Table 1. Correlation of PRDM5 in GC and clinical parameters in 162 cases

\begin{tabular}{|c|c|c|c|}
\hline & All patients $(n=162)$ & $\begin{array}{l}\text { Low expression of } \\
\text { PRDM5 }\end{array}$ & P value \\
\hline Age & & & 0.685 \\
\hline$\leq 60$ & $76(46.9 \%)$ & $59(77.6 \%)$ & \\
\hline$>60$ & $86(53.1 \%)$ & $69(80.2 \%)$ & \\
\hline Gender & & & 0.902 \\
\hline Female & $51(31.5 \%)$ & $40(78.4 \%)$ & \\
\hline Male & $111(68.5 \%)$ & $88(79.3 \%)$ & \\
\hline Lauren classification & & & 0.524 \\
\hline Intestinal type & $34(21.0 \%)$ & $26(76.5 \%)$ & \\
\hline Mixed type & $46(28.4 \%)$ & $39(84.8 \%)$ & \\
\hline Diffuse type & $82(50.6 \%)$ & $63(76.8 \%)$ & \\
\hline Differentiation & & & 0.713 \\
\hline Well differentiated & $76(46.9 \%)$ & $61(80.3 \%)$ & \\
\hline Poorly differentiated & $86(53.1 \%)$ & $67(77.9 \%)$ & \\
\hline Tumor diameter & & & 0.919 \\
\hline$\leq 5 \mathrm{~cm}$ & $118(72.8 \%)$ & $93(78.8 \%)$ & \\
\hline$>5 \mathrm{~cm}$ & $44(27.2 \%)$ & $35(79.5 \%)$ & \\
\hline pT classification & & & 0.229 \\
\hline $\mathrm{T} 1 \sim 3$ & $110(67.9 \%)$ & $84(76.4 \%)$ & \\
\hline $\mathrm{T} 4$ & $52(32.1 \%)$ & $44(84.6 \%)$ & \\
\hline pN classification & & & 0.317 \\
\hline N0 & $55(34.0 \%)$ & $41(74.5 \%)$ & \\
\hline $\mathrm{N} 1 \sim 3$ & $107(66.0 \%)$ & $87(81.3 \%)$ & \\
\hline pM classification & & & 0.580 \\
\hline Absent & $158(97.5 \%)$ & $124(78.5 \%)$ & \\
\hline Present & $4(2.5 \%)$ & $4(100.0 \%)$ & \\
\hline Vascular invasion & & & 0.590 \\
\hline Absent & $113(69.8 \%)$ & $88(77.9 \%)$ & \\
\hline Present & $49(30.2 \%)$ & $40(81.6 \%)$ & \\
\hline Nerve invasion & & & 0.503 \\
\hline Absent & $117(72.2 \%)$ & $94(80.3 \%)$ & \\
\hline Present & $45(27.8 \%)$ & $34(75.6 \%)$ & \\
\hline Clinical staging & & & $0.036^{*}$ \\
\hline $\mathrm{I}+\mathrm{II}$ & $79(48.8 \%)$ & $57(72.2 \%)$ & \\
\hline III+IV & $83(51.2 \%)$ & $71(85.5 \%)$ & \\
\hline
\end{tabular}

$* \mathrm{P}<0.05$. 
A
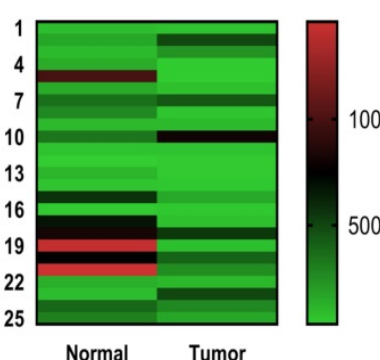

C

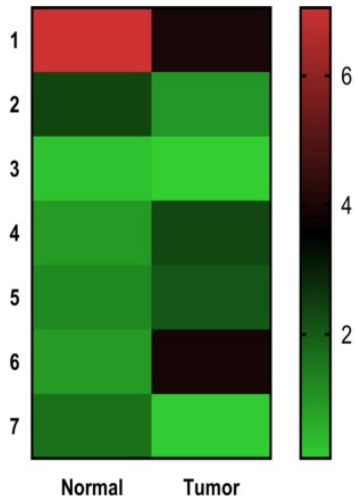

500
B

1000

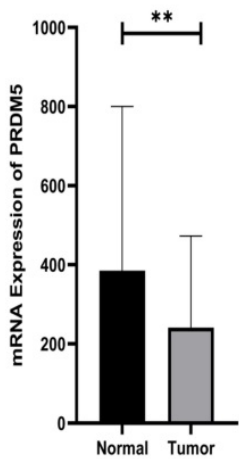

$\begin{array}{llllllll}\mathrm{T} 1 & \mathrm{~N} 1 & \mathrm{~T} 2 & \mathrm{~N} 2 & \mathrm{~T} 3 & \mathrm{~N} 3 & \mathrm{~T} 4 & \mathrm{~N} 4\end{array}$

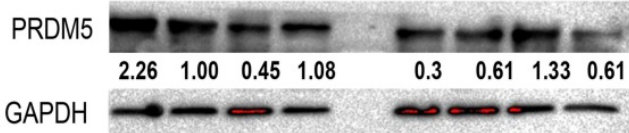

$\begin{array}{lllllllll}\text { T5 } & \text { N5 } & \text { T6 } & \text { N6 } & \text { T7 } & \text { N7 } & \text { T8 } & \text { N8 }\end{array}$

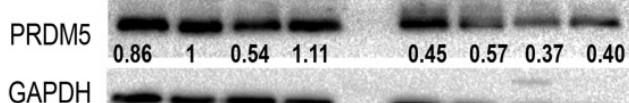

GAPDH

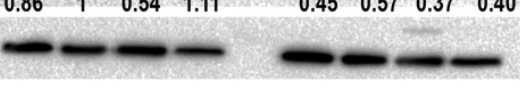

Figure 1. PRDM5 mRNA expression and protein expression are downregulated in GC. A-B Expression of PRDM5 was frequently downregulated in gastric tumor tissues (tumor) compared with adjacent or normal gastric tissue samples (normal) in public databases. C-D mRNA levels of PRDM5 detected by Q-rtPCR and protein levels of PRDM5 detected by western blot in tumors and adjacent tumors. Data are shown as mean \pm SD $* p<0.05 ; * * p<0.01 ; * * * p<0.001$, ***** $<0.0001$.
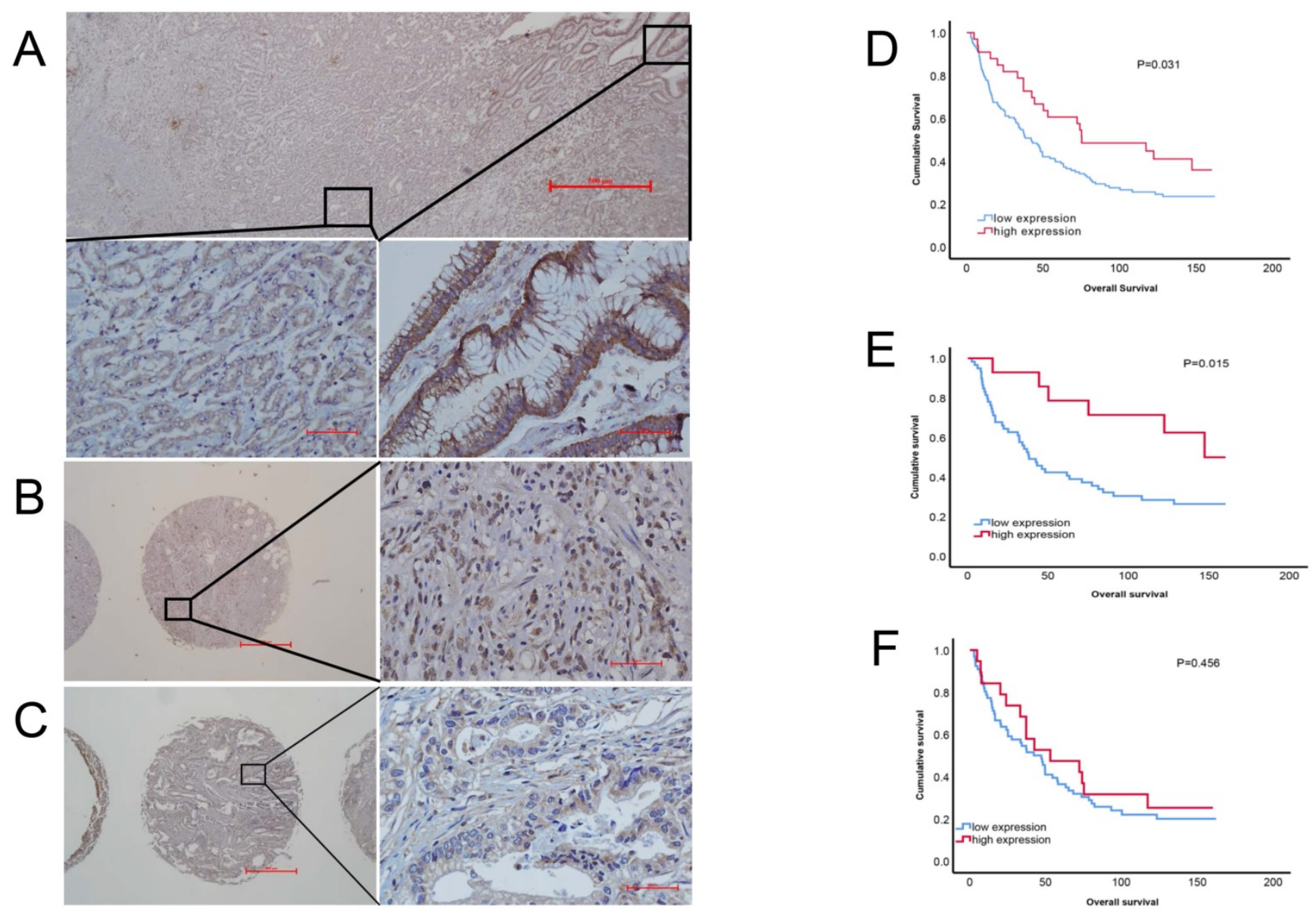

Figure 2. Protein expression of PRDM5 in GC tissues ( $\times \mathbf{4 0 / \times 4 0 0 ~ o p t i c a l ~ m a g n i f i c a t i o n ) . ~ A . ~ P R D M 5 ~ e x p r e s s i o n ~ i n ~ c a r c i n o m a ~ a n d ~ p a r a c a n c e r o u s ~ t i s s u e s ~ o f ~ a ~ p a t i e n t ~}$ with GC. B-C. The typical examples of high and low expression in GC tissues, respectively. Original magnification $\times 400$. D-F. Kaplan-Meier curves showed that PRDM5 expression is significantly associated with prolonged survival in all GC patients (D) and in patients with well differentiated (E) or poor differentiated (F). 
Table 2. Univariate and multivariate Cox proportional hazard analyses for cancer-specific survival

\begin{tabular}{lllll}
\hline Overall survival & \multicolumn{3}{l}{ Univariable } & \multicolumn{2}{l}{ Multivariable } \\
\cline { 2 - 5 } & Hazard ratio & $\mathrm{P}$ & Hazard ratio & $\mathrm{P}$ \\
\hline $\begin{array}{l}\text { Differentiation } \\
\text { (well versus poor) }\end{array}$ & $0.755(0.534-1.068)$ & 0.112 & $0.931(0.633-1.370)$ & 0.717 \\
Vascular invasion & $1.449(1.004-2.092)$ & 0.048 & $1.156(0.758-1.7622)$ & 0.501 \\
$\begin{array}{l}\text { Nerve invasion } \\
\text { Tumor diameter } \\
(>5 \text { cm versus } \leq 5 \mathrm{~cm})\end{array}$ & $1.371(0.937-2.006)$ & 0.104 & $1.193(0.772-1.845)$ & 0.427 \\
$\begin{array}{l}\text { Clinical staging (III+IV } \\
\text { versus I+II) }\end{array}$ & $2.725(1.909-3.888)$ & $<0.001^{*}$ & $2.377(1.587-3.561)$ & $<0.001^{*}$ \\
$\begin{array}{l}\text { PRDM5 expression } \\
\text { (high versus low) }\end{array}$ & $0.592(0.365-0.960)$ & $0.034^{*}$ & $0.602(0.369-0.984)$ & $0.043^{*}$ \\
\hline *P $<0.05$. & & & & \\
\hline
\end{tabular}

\section{PRDM5 inhibited the proliferation and migration of GC cells}

To explore the role of PRDM5 in the development of GC, we conducted further experiments in GC cells. Firstly, we examined the basic expression of PRDM5 in three cell lines by
Western blot. We found that the expression of PRDM5 in HGC-27 was significantly silenced. Next, we knocked down PRDM5 in AGS and SGC-7901 cell lines, and overexpressed PRDM5 in HGC-27 cell lines. For better quality control, gastric epithelial cell GES-1 and lung adenocarcinoma cell A549 were selected for the experiment. In A549 cell line, PRDM5 has been shown to be silenced by previous studies.Not surprisingly, we found that PRDM5 knockdown GC cells (AGS, SGC7901) significantly enhanced their proliferation ability (Figure 3A-B) and the ectopic expression of PRDM5 inhibited the proliferation of tumor cells (Figure 3D-E). After knockdown of PRDM5, the cloning and migration ability of GC cells were significantly improved (Figure 3F-H). However, after the exogenous overexpression of PRDM5 in A549, the cell migration ability was inhibited. In gastric epithelial cells GES-1, PRDM5 knockdown also significantly improved the cell proliferation, cloning and migration ability (Figure 3C, F \& H).

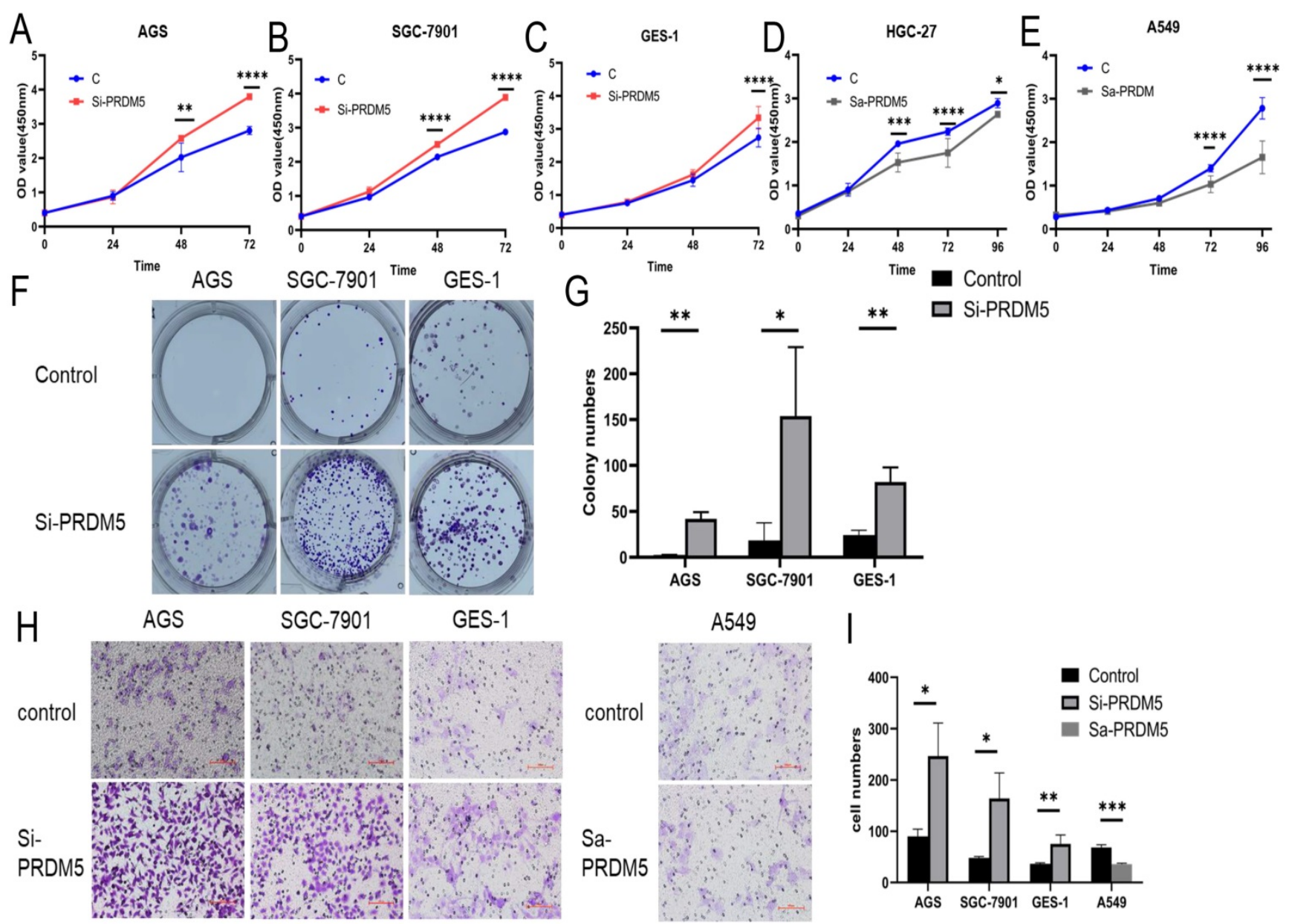

Figure 3. PRDM5 inhibited the proliferation and migration of GC cells. A-E. The proliferation ability of GC cells was inhibited by PRDM5. F. The proliferation ability of cells was enhanced after PRDM5 knockdown. G. Statistical results of cell cloning experiments. H. Cell migration assay after changing PRDM5 expression. I. Statistical results of cell migration assay. 

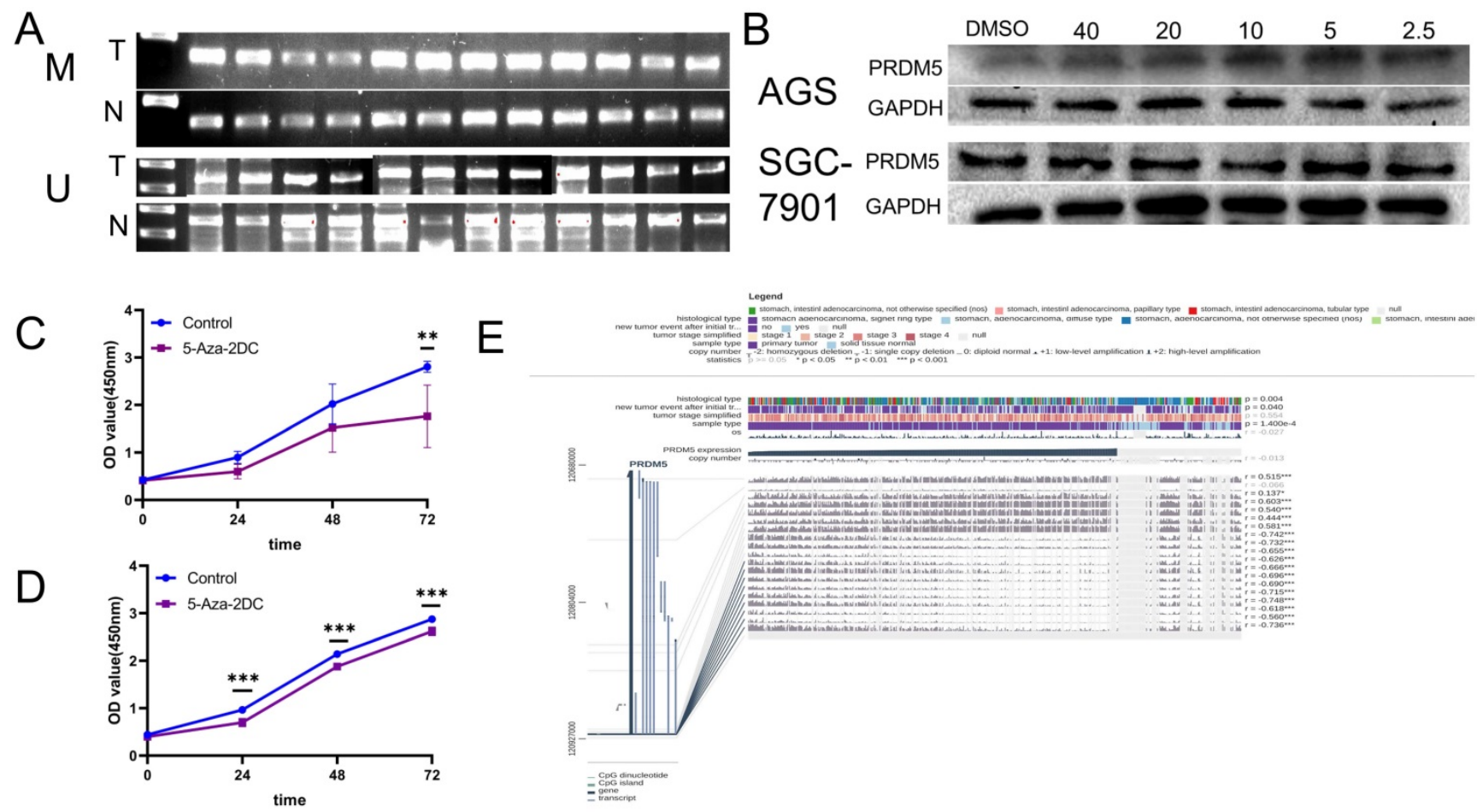

Figure 4. The promoter of PRDM5 is often methylated. A. The methylated specific primers and unmethylated specific primers were used to detect the difference of methylation between tumor and normal tissues. M primers specific to methylated template DNA, $U$ primers specific to unmethylated template DNA, T tumor, $N$ normal. B. PRDM5 protein expression in cells treated with methylation inhibitors at different concentrations ( $\mu$ M). C-D. Proliferation ability of AGS(C) and SGC-7901 (D) cells which were treated with methylation inhibitors at appropriate concentrations. E. MEXPRESS analysis about the correlation between DNA methylation of CPG islands in the $5^{\prime}$ promoter region and expression level of PRDM5.

\section{The low expression of PRDM5 is related to promoter methylation}

Previous studies have shown that PRDM5 is silenced in tumors due to methylation of its promoter [5]. Firstly, MSP was detected in fresh GC tissues and paired adjacent normal tissues from 12 patients. We found that methylated bands could be detected in all carcinomas, but rarely in the paired adjacent normal tissues (Figure 4A). Further, we verified the methylation mechanism of PRDM5 in GC cells. By treating GC cells with different concentrations of methylation inhibitors (5-Aza-2DC), we found that suitable concentrations of 5-Aza-2DC could restore the expression of PRDM5 (Figure 4B). Next, we continued to verify the proliferation ability of GC cells after the addition of inhibitors. We found that the proliferation activity of GC cell lines was inhibited when treated with a certain concentration of inhibitor that could restore PRDM5 protein expression (Figure $4 C, D)$. Finally, we used MEXPRESS to analyze the correlation between DNA methylation of CpG islands in the $5^{\prime}$ promoter region and expression level of PRDM5. The samples shown in Figure 4E are ordered by expression value. The expression of PRDM5 in GC negatively correlated with the level of DNA methylation in the promoter, which was confirmed by Pearson correlation coefficients ( $\mathrm{r}$ up to $-0.748, p<$
0.001). MEXPRESS analysis also revealed that PRDM5 mRNA expression level was much lower in GC samples than those in control samples $(p=1.400 \mathrm{e}-4)$. Moreover, we found that the methylation of PRDM5 promoter was closely related to the histopathological types of patients and the recurrence and progression of tumors. Thus, these data demonstrate that promoter DNA hypermethylation of PRDM5 may result in downregulation of its mRNA expression, which, to some extent, supports our preliminary study results.

\section{Discussion}

Gastric cancer is the fifth most common cancer and the third leading cause of cancer death in the world, with the highest incidence in East Asia [12]. High-income regions in Asia Pacific and East Asia have the highest age-standardized incidence rates, with nearly half of global cases occurring in China [13] .This urges us to understand GC through more research, so as to alleviate or even cure GC and reduce the disease burden. Early GC can usually be cured by endoscopic resection or radical surgery, but the prognosis of advanced GC is still poor, despite chemotherapy, radiotherapy, targeted therapy, immunotherapy and other therapeutic methods. So, there are many trials going on that combine immunotherapy, chemotherapy and surgery for local 
GC [14]. On this basis, we are still actively looking for new breakthroughs in order to help patients with GC.

Genetic and epigenetic changes can participate in the occurrence and development of GC through activation of growth-promoting pathways and inactivation of tumor suppressor pathways. Moreover, in recent comprehensive studies of genetic and epigenetic changes in GC, epigenetic changes such as activation of Wnt pathways, inactivation of cell cycle regulation, and damage to mismatch repair have been more frequent than genetic changes [15]. In epigenetic changes, abnormal DNA methylation of a promoter CpG Island (CGI) can continuously inhibit the transcription of its downstream genes, and tumor suppressor genes can be inactivated by this mechanism [16]. The DNA demethylation drugs 5-azacytidine (azacitidine) and 5-aza-2 '-deoxycytidine (decitabine) that are clinically used in patients with myelodysplastic syndrome can restore abnormal DNA methylation [17]. Multiple clinical trials involving the use of demethylating agents for solid tumors are currently under way. Several trials have shown that demethylating agents can increase chemotherapeutic sensitivity in some drug-resistant ovarian and lung cancers $[18,19]$. In GC, studies have verified that methylation agents have anti-tumor effects on SN38 and CDDP resistant GC cell lines [20]. However, the exact epigenetic mechanism is still unclear. Furthermore, we tried to explore tumor suppressor factors that activate this mechanism.

PRDM5 is considered to have tumor suppressive activity because its promoter contains a CpG island, which is highly methylated in tumors, leading to silencing of its expression in a variety of tumors [7]. Our study shows that PRDM5 is generally downregulated in GC, which is consistent with previous studies [8]. Based on the analysis of GC data from the TCGA database, we found that the mRNA level of PRDM5 was down-regulated. And we found that the protein expression of PRDM5 was generally inhibited in tumor tissues. Due to the large heterogeneity of tumors, we further detected PRDM5 protein expression in 162 cases of GC in tissue microarray. It was found that the expression of PRDM5 was negatively correlated with clinical stage. Based on these results, we wondered whether PRDM5 could predict the prognosis of patients with GC, which has not been studied before. Our results showed that patients with high PRDM5 expression had a significantly better prognosis, especially in patients with well differentiated GC. Through univariate and multivariate regression analysis, we found that PRDM5 expression is the same as clinical stage and tumor size, which are independent prognostic factors of GC. These results suggest that
PRDM5 plays an important role in the treatment of advanced GC, which means that we may be able to find new therapeutic targets in the treatment of advanced GC. Therefore, we further explored the mechanism of PRDM5 in GC. As we have seen, the proliferation activity and migration ability of GC cells were increased after knockdown of PRDM5 expression, while the proliferation activity was inhibited after overexpression of PRDM5. Then, we found that PRDM5 expression was increased more or less in cells treated with methylation inhibitors. We were curious to see if methylation of the PRDM5 promoter had any clinical significance. We performed an analysis of the gene promoter methylation in a public database. We found the expression of PRDM5 in GC negatively correlated with the level of DNA methylation in the promoter. Moreover, we found that the methylation of PRDM5 promoter was closely related to the histopathological types of patients and the recurrence and progression of tumors. Thus, these data demonstrate that promoter DNA hypermethylation of PRDM5 may result in downregulation of its mRNA expression, which, to some extent, supports our preliminary study results.

Previous studies have demonstrated that promoter reporting activity of cyclin D1 (CCND1), a target gene downstream of the Wnt/ $\beta$-catenin signaling pathway, is significantly reduced when PRDM5 is overexpressed.Chromatin immunoprecipitation (ChIP) assay showed that PRDM5 can directly bind to the promoters of several oncogenes such as CDK4 and Twist1.Overexpression of PRDM5 significantly reduced the levels of active transcriptional markers $\mathrm{H} 3 \mathrm{~K} 4 \mathrm{me} 3$ and acetylated histone $\mathrm{H} 4$ of CDK4, Twist1 promoter.In addition, many other genes are regulated by PRDM5, including oncogenes such as p53, MYC and MDM2 [5]. In gastric cancer, whether PRDM5 plays a role in tumor inhibition through these possible pathways still needs further verification. It is worth noting that although PRDM5 expression is reduced in most tumors, such as lung cancer, cervical cancer, prostate cancer, glioma and other tumors [21-24]. However, there are exceptions. In melanoma and acute myelogenous leukemia, PRDM5 is overexpressed in tumor cells and is believed to promote tumor progression through activation of the JNK pathway [25, 26]. This opposite regulatory function may be due to its special role in transcriptional regulation. Studies have claimed that it can promote and inhibit transcriptional genes according to promoters and cell types [27].

Our study suggests that methylation of PRDM5 promoter plays an indispensable role in GC. However, we still need more representative clinical samples to support our results because of tumor 
heterogeneity. It is well known that abnormal methylation plays a significant role in the development of GC because Helicobacter pylori infection leads to abnormal methylation [28]. Therefore, we should include more clinical relevant factors, such as the history of Helicobacter pylori infection, in the follow-up of patients, which would make our results more convincing. In terms of mechanism, it is far from enough to understand the role of PRDM5 as a tumor suppressor in GC. Activated growth-promoting pathways in gastric cancer include Wnt, Akt/mTOR and mitogenactivated protein kinase (MAPK) pathways. These pathways can be activated not only by activating oncogene mutations, but also by inactivating their negative regulators. PRDM5 antagonizes the Wnt/ $\beta$-catenin signaling pathway in both normal and tumor cells [5]. However, GC is a complex and systemic disease, and how PRDM5 plays a role in vivo is still unknown. However, GC is a complex and systemic disease, and there are still many questions about how PRDM5 plays its role in the body, such as why the promoter of PRDM5 is methylated and how it is methylated.More in vivo studies are needed to answer these questions.

In conclusion, our results found that PRDM5 plays an important role in the development of GC. Especially in advanced GC, it has good prognostic significance.

\section{Conclusion}

In this study, we demonstrated that PRDM5 plays a role as a tumor suppressor in GC, and its down-regulation in GC patients is partly caused by DNA hypermethylation. More importantly, we found that PRDM5 is a potential prognostic factor, especially in well-differentiated GC, where high expression of PRDM5 suggests a better outcome.

\section{Acknowledgements}

The work was supported by grants from Natural Science Foundation of Jiangsu Province (BK20191208), Nantong Science and Technology Foundation grant (JC2018031, JCZ19041, JCZ20205), Nantong Health and Care Foundation grant (MB2020022, MA2020009).

\section{Competing Interests}

The authors have declared that no competing interest exists.

\section{References}

1. Corso G, Velho S, Paredes J, Pedrazzani C, Martins D, Milanezi F, et al. Oncogenic mutations in gastric cancer with microsatellite instability. European Journal of Cancer. 2011; 47: 443-51.
2. Shi J, Yao D, Liu W, Wang N, Lv H, He N, et al. Frequent gene amplification predicts poor prognosis in gastric cancer. Int J Mol Sci. 2012; 13: 4714-26.

3. Calcagno DQ, Gigek CO, Chen ES, Burbano RR, Smith Mde A. DNA and histone methylation in gastric carcinogenesis. World J Gastroenterol. 2013; 19: 1182-92.

4. Andrew P. Feinberg BV. Hypomethylation distinguishes genes of some human cancers from their normal counterparts. NATURE. 1983; 301: 4.

5. Shu XS, Geng H, Li L, Ying J, Ma C, Wang Y, et al. The epigenetic modifier PRDM5 functions as a tumor suppressor through modulating WNT/beta-

catenin signaling and is frequently silenced in multiple tumors. PLoS One. 2011; 6: e27346.

6. Shivapurkar N, Maitra A, Milchgrub S, Gazdar AF. Deletions of chromosome 4 occur early during the pathogenesis of colorectal carcinoma. Hum Pathol. 2001; 32: 169-77.

7. Deng Q, Huang S. PRDM5 is silenced in human cancers and has growth suppressive activities. Oncogene. 2004; 23: 4903-10.

8. Watanabe Y, Toyota M, Kondo Y, Suzuki H, Imai T, Ohe-Toyota M, et al. PRDM5 identified as a target of epigenetic silencing in colorectal and gastric cancer. Clin Cancer Res. 2007; 13: 4786-94.

9. Watanabe Y, Kim HS, Castoro RJ, Chung W, Estecio MR, Kondo K, et al. Sensitive and specific detection of early gastric cancer with DNA methylation analysis of gastric washes. Gastroenterology. 2009; 136: 2149-58.

10. Koch A, De Meyer T, Jeschke J, Van Criekinge W. MEXPRESS: visualizing expression, DNA methylation and clinical TCGA data. BMC Genomics. 2015; 16: 636.

11. Chen Y, Zhang Y, Tan Y, Liu Z. Clinical significance of SPARC in esophageal squamous cell carcinoma. Biochem Biophys Res Commun. 2017; 492: 184-91.

12. Bray F, Ferlay J, Soerjomataram I, Siegel RL, Torre LA, Jemal A. Global cancer statistics 2018: GLOBOCAN estimates of incidence and mortality worldwide for 36 cancers in 185 countries. CA Cancer J Clin. 2018; 68: 394-424.

13. Collaborators GBDSC. The global, regional, and national burden of stomach cancer in 195 countries, 1990-2017: a systematic analysis for the Global Burden of Disease study 2017. Lancet Gastroenterol Hepatol. 2020; 5: 42-54.

14. Kataoka K, Deleersnijder A, Lordick F. Will molecular target agents enable the multidisciplinary treatment in stage IV gastric cancer? Eur J Surg Oncol. 2017; 43: 1835-45.

15. Yoda Y, Takeshima H, Niwa T, Kim JG, Ando T, Kushima R, et al. Integrated analysis of cancer-related pathways affected by genetic and epigenetic alterations in gastric cancer. Gastric Cancer. 2015; 18: 65-76.

16. Ushijima T. Detection and interpretation of altered methylation patterns in cancer cells. NATURE REVIEWS 2005; 5: 8.

17. Kantarjian H, Issa JP, Rosenfeld CS, Bennett JM, Albitar M, DiPersio J, et al. Decitabine improves patient outcomes in myelodysplastic syndromes: results of a phase III randomized study. Cancer. 2006; 106: 1794-803.

18. Juergens RA, Wrangle J, Vendetti FP, Murphy SC, Zhao M, Coleman B, et al. Combination epigenetic therapy has efficacy in patients with refractory advanced non-small cell lung cancer. Cancer Discov. 2011; 1: 598-607.

19. Matei D, Fang F, Shen C, Schilder J, Arnold A, Zeng Y, et al. Epigenetic resensitization to platinum in ovarian cancer. Cancer Res. 2012; 72: 2197-205.

20. Moro H, Hattori N, Nakamura Y, Kimura K, Imai T, Maeda M, et al. Epigenetic priming sensitizes gastric cancer cells to irinotecan and cisplatin by restoring multiple pathways. Gastric Cancer. 2020; 23: 105-15.

21. Haiyan Cheng JM, Ziwei Feng, Kejin Huang, Yuxia Wang, Yunyan Zhang. Study on the role of tumor suppressor gene PRDM5 in the development of cervical squamous cell carcinoma induced by HPV16 virus. Prac Oncol J. 2018; 32.

22. Wang X, Chang H, Gao G, Su B, Deng Q, Zhou H, et al. Silencing of PRDM5 increases cell proliferation and inhibits cell apoptosis in glioma. Int J Neurosci. 2020: 1-10.

23. Yang Wang ZXMHYS. PRDM5 gene inhibits 22RV1 growth in prostate cancer cells. Academic Journal of Second Military Medical University. 2016; 37: 724-8.

24. Tan S-X, Hu R-C, Tan Y-L, Liu J-J, Liu W-E. Promoter methylation-mediated downregulation of PRDM5 contributes to the development of lung squamous cell carcinoma. Tumor Biology. 2014; 35 : 4509-16.

25. Wang L, Ding QQ, Gao SS, Yang HJ, Wang M, Shi Y, et al. PRDM5 promotes the proliferation and invasion of murine melanoma cells through up-regulating JNK expression. Cancer Med. 2016; 5: 2558-66. 
26. Zhou P, Chen X, Li M, Sun X, Tan J, Wang X, et al. Overexpression of PRDM5 promotes acute myeloid leukemia cell proliferation and migration by activating the JNK pathway. Cancer Med. 2019; 8: 3905-17.

27. Duan Z, Person RE, Lee HH, Huang S, Donadieu J, Badolato R, et al. Epigenetic Regulation of Protein-Coding and MicroRNA Genes by the Gfi1-Interacting Tumor Suppressor PRDM5. Molecular and Cellular Biology. 2007; 27: 6889-902.

28. Maekita T, Nakazawa K, Mihara M, Nakajima T, Yanaoka K, Iguchi M, et al. High levels of aberrant DNA methylation in Helicobacter pylori-infected gastric mucosae and its possible association with gastric cancer risk. Clin Cancer Res. 2006; 12: 989-95. 